15

\title{
Роль матричного эффекта в анализах биологических объектов масс-спектрометром с индуктивно связанной плазмой (ICP-MS)
}

\author{
(C) Т.К. Нурубейли, К.З. Нуриев, З.К. Нурубейли, К.Б. Гурбанов
}

Институт фозики НАН Азербайджана, Az-1143 Баку, Азербайджан

e-mail: kamilnuri@rambler.ru

(Поступило в Редакцию 2 августа 2017 г. В окончательной редакции 17 сентября 2018 г.)

На примере ряда элементов в цельной крови и моче с использованием ICP-MS с квадрупольным анализатором Agilent-7700 произведена попытка устранения влияния не спектрального матричного эффекта, приводящая к подавлению интенсивности аналитического сигнала на выходе прибора. Показано, что основную роль в появлении не спектрального матричного эффекта играют как кислотный и солевой составы, так и органические вещества, входящие в состав самого образца. Оценено влияние кислотного, солевого и органического составов матрицы цельной крови и мочи на занижение результатов определения ряда элементов. Показано также, что внутренний стандарт должен выбираться исходя из близости первого потенциала его ионизации к первому потенциалу ионизации определяемого элемента. Исследованы и оценены вклады не спектральных матричных эффектов в искажение результатов анализа биологических жидкостей, установлено, что зависимость матричного эффекта от солей и кислотностей матрицы, а также от режима работы прибора, носит аддитивный характер.

DOI: $10.21883 / J T F .2019 .06 .47745 .2451$

\section{Введение}

Масс-спектрометрию с индуктивно связанной плазмой (ICP-MS) достаточно давно начали использовать в лабораториях, занимающихся анализом биологических объектов, а также разработкой новых лекарственных препаратов с целью изучения роли химических элементов в жизнедеятельности человека [1,2]. До недавнего времени массовое использование ее было ограничено ввиду дороговизны и сложности оборудования. На сегодняшний день ICP-MS занимает лидирующие позиции в этой области. Последнее связано, с одной стороны, с большей доступностью и производительностью метода, и, с другой, с рекордно низким пределом обнаружения химических элементов и их изотопов, что требуется при исследованиях аналитов с очень низкими концентрациями в образце. При этом немаловажными фактами являются возможности проведения этим методом многоэлементного анализа в широком (до 10 порядков) динамическом диапазоне градуировочной зависимости [3]. Последнее обстоятельство особенно важно для таких образцов, как кровь, слюна и волосы человека, в виду необходимости одновременного определения из одной пробы элементов с низким и высоким содержанием, а объем такого рода материалов обычно мал. С другой стороны, как и любой инструментальный метод, массспектрометрия (в том числе ICP-MS) имеет свои недостатки, важнейшими из которых являются спектральные и неспектральные матричные эффекты $[1,4]$.

К спектральным относятся наложение ионов с одинаковыми $M / q$, приводящее к искажению масс-спектра, а не спектральные эффекты связаны с дрейфом чувствительности, обусловленным как составом и методом пробоподготовки, так и изменением характеристики прибора.
Зачастую в масс-спектрометрии с ИСП требуется определить микропримеси на фоне высоких концентраций основного вещества (матрицы). При анализе растворов с высокими содержаниями матрицы (более $0.5-1.0 \mathrm{~g} / \mathrm{l})[5,6]$ может иметь место значительное (до $50 \%$ и более) понижение чувствительности прибора за счет матричного эффекта (МЭ).

МЭ в наибольшей степени влияет на легкие изотопы с массами до 80 a.m.u. [7,8]. В общем случае чем выше атомная масса элементов, тем заметнее становится матричный эффект. Изменение чувствительности прибора, связанное с матричным эффектом, можно компенсировать, например, введением внутреннего стандарта.

В связи с этим необходимо отметить, что при анализе биологических объектов могут проявляться неспектральные матричные эффекты под влиянием как кислоты (прежде всего азотной), используемой при минерализации, так и органических растворителей и составных компонентов биологических жидкостей. Влияние кислоты на результаты анализа заключается чаще всего в подавлении аналитических сигналов на выходе и зависит как от концентрации, так и от природы кислоты [9-11].

Настоящая работа посвящена исследованию возможных влияний матрицы на результаты измерения и поиску более простых способов их устранения за счет варьирования инструментальных настроек масс-спектрометра, оптимизации методик измерения и обработки аналитических сигналов. В качестве аналитов в работе были выбраны $\mathrm{Mn}^{-}, \mathrm{Co}^{-}, \mathrm{Cu}^{-}, \mathrm{Zn}^{-}, \mathrm{Ga}^{-}, \mathrm{As}^{-}, \mathrm{Se}^{-}, \mathrm{Cd}^{-}$, $\mathrm{Pt}^{-}, \mathrm{Pb}$-элементы, выбор которых продиктован задачами, стоящими перед медиками при разработке биологических методик для анализа биологических жидкостей и лекарственных препаратов. 


\section{Теоретическая часть}

В настоящее время для плазменных масс-спектрометров, в том числе ИСП-МС, центральной является проблема получения надежных количественных данных. Особенно это касается анализа биологических объектов и фармацевтических препаратов, так как в большинстве случаев соотношение пиков измеренного масс-спектра не соответствует истинному составу пробы. Причинами этого являются неодинаковый выход различных элементов, что связано как с особенностями процесса ионообразования в плазме, так и дискриминациями их по массе в системе разделения и регистрации $[12,13]$.

Для учета этого несоответствия еще в 70-х годах прошлого столетия, использовали так называемый внутренний стандарт (internal standard, IS) - элемент (элементы) с известным количеством и составом, вводимый в анализируемый образец. При этом количество исследуемых элементов определяется относительно IS, а так называемый коэффициент относительной чувствительности (relative sensitivity coefficient, RSC) вычисляется по выражению [14-16]

$$
\mathrm{RSC}_{\mathrm{IS}}^{x}=\frac{J_{x}}{J_{\mathrm{IS}}} \frac{n_{\mathrm{IS}}}{n_{x}}
$$

где $J_{x}$ и $J_{\mathrm{IS}}-$ аналитические сигналы определяемого элемента $x$ и внутреннего стандарта (обычно ими служат интенсивности токов однозарядных ионов); $n_{x}$ и $n_{\mathrm{IS}}$ - концентрации этих элементов в пробе соответственно.

Для количественного определения $\mathrm{RSC}_{\mathrm{IS}}^{x}$ применяют термодинамический подход, учитывающий в расчетах термодинамические характеристики индивидуальных веществ [17]. Суть его заключается в определении экспериментальных значений RSC и установлении корреляции с основными физико-химическими свойствами определяемых элементов. Такая корреляция позволяет выделить основные процессы в плазме, ответственные за образование ионов, и применить к ним плазменный или кинетический подходы [14].

Расчет эффективности ионизации однократно заряженных атомных ионов элементов $x$ и IS может быть проведен с использованием уравнения Саха [14]

$$
\begin{aligned}
g_{x}= & \frac{\alpha_{x}}{1-\alpha_{x}}=\frac{\left(2 \pi m_{e} k T_{u}\right)^{3 / 2}}{n_{e} h^{3}} \frac{2 Z_{x}^{+}}{Z_{x}^{0}} \exp \left(-\frac{\varphi_{x}^{+}}{k T_{u}}\right) \\
& \times \exp \left(-\frac{B_{x}}{k T_{a}}\right), \\
g_{\mathrm{IS}}= & \frac{\alpha_{\mathrm{IS}}}{1-\alpha_{\mathrm{IS}}}=\frac{\left(2 \pi m_{e} k T_{u}\right)^{3 / 2}}{n_{e} h^{3}} \frac{2 Z_{\mathrm{IS}}^{+}}{Z_{\mathrm{IS}}^{0}} \exp \left(-\frac{\varphi_{\mathrm{IS}}^{+}}{k T_{u}}\right) \\
& \times \exp \left(-\frac{B_{\mathrm{IS}}}{k T_{a}}\right),
\end{aligned}
$$

где $\alpha_{x}$ и $\alpha_{\mathrm{IS}}-$ степень ионизации атомов элемента $x$ и внутреннего стандарта, $n_{e}-$ плотность электронов, $m$ - масса электрона, $\varphi_{x}^{+}$и $\varphi_{\mathrm{IS}}^{+}-$потенциалы первичных ионизаций элементов $x$ и IS, $B_{x}, B_{\mathrm{IS}}-$ энергии атомизации этих элементов, $k$ - постоянная Больцмана, $h$ - постоянная Планка, $T_{a}$ и $T_{u}$ - модельные температуры атомизации (диссоциации) и ионизации элементов $x$ и IS.

Приняв за $\mathrm{RSC}_{\mathrm{IS}}^{x}$ отношение степеней ионизации атомов $x$ (2) и внутреннего стандарта (3), получаем [14]

$$
\mathrm{RSC}_{\mathrm{IS}}^{x}=\exp \left(-\frac{B_{x}-B_{\mathrm{IS}}}{k T_{a}}\right) \exp \left(-\frac{\varphi_{x}^{+}-\varphi_{\mathrm{IS}}^{+}}{k T_{u}}\right) F_{\text {instr }},
$$

где $F_{\text {instr }}$ - приборный фактор, зависящий от отношения атомных масс $M$ элементов $x$ и IS и имеющий вид

$$
F_{\text {instr }}=\left(\frac{M_{x}}{M_{\mathrm{IS}}}\right)^{\gamma} \text {. }
$$

Для других плазменных источников ионов можно подобрать модельные температуры $T_{a}$ и $T_{u}$, обеспечивающие соответствие между экспериментальными и рассчитанными по (5) значениями $\mathrm{RSC}_{\mathrm{IS}}^{x}$.

Отметим, что в условиях эксперимента реализуемая в ИСП-МС зависимость между экспериментальным значением $\mathrm{RSC}_{\mathrm{IS}}^{x}$ и энергией атомизации (диссоциации) атомов $\left(B_{x}, B_{\mathrm{IS}}\right)$ не обнаруживалась, коэффициент корреляции не превышал 0.03. Это свидетельствует об отсутствии селективности атомизации, которая может приводить к различиям элементного состава газовой фазы и исходного раствора. Действительно, как показано в [13], в случае ИСП-МС процессы диссоциации и ионизации атомов элементов разделены в пространстве и во времени. Это позволяет упростить формулу (4)

$$
\mathrm{RSC}_{\mathrm{IS}}^{x}=\exp \left(\frac{\varphi_{x}^{+}-\varphi_{\mathrm{IS}}^{+}}{k T_{u}}\right)\left(\frac{m_{x}}{m_{\mathrm{IS}}}\right)^{\gamma}
$$

В табл. 1 даны значения $\mathrm{RSC}_{\mathrm{IS}}^{x}$, определенные и рассчитанные с использованием параметров $T_{u}$ и $\gamma$, для ряда элементов. Показано, что средние относительные отклонения этих значений от экспериментальных величин достаточно существенны $(\Delta R=0.42)$. Коэффициент корреляции между логарифмами экспериментальных значений $\mathrm{RSC}_{\mathrm{IS}}^{x}$ и потенциалами ионизации составляет 0.51. При этом $T_{u}$ оказывается намного выше температуры в центральной области индукционной плазмы почти на порядок.

Приведенные в табл. 1 данные показывают, что функциональная зависимость $\mathrm{RSC}_{\mathrm{IS}}^{x}$ от потенциала ионизации и массы ионов в случае с индуктивно связанной плазмой не достаточно точно описывает процесс образования ионов, в отличие от лазерной и искровой плазм. Это свидетельствует о возможном наличии какого-то фактоpa, влияющего на процесс ионообразования в ICP-MS. Отметим, что в [18] была обнаружена корреляция RSC со сродством к электрону и электроотрицательностью атома.

Пробоподготовка. Основным этапом в масс-спектрометрическом исследовании биологических образцов является пробоподготовка. В настоящее время для этого 
Таблица 1. Потенциалы атомизации $\left(\varphi_{x^{0}}\right)$, потенциалы однократной ионизации $\left(\varphi_{x}^{+}\right)$, атомные массы $M_{x}$, рассчитанные $\left(\mathrm{RSC}_{\mathrm{IS}}^{x, \text { theor }}\right)$ и экспериментальные $\mathrm{RSC}_{\mathrm{IS}}^{x, \text { exper }}$ и отношение теоретически рассчитанного $\mathrm{RSC}$ к экспериментально измеренному $(\Delta R)$

\begin{tabular}{c|c|c|c|c|c|c|c|c|c|c|c}
\hline & $\mathrm{P}$ & $\mathrm{Mn}$ & $\mathrm{Co}$ & $\mathrm{Cu}$ & $\mathrm{Zn}$ & $\mathrm{Ga}$ & $\mathrm{As}$ & $\mathrm{Se}$ & $\mathrm{Cd}$ & $\mathrm{Pt}$ & $\mathrm{Pb}$ \\
\hline$\varphi_{x}^{0}, \mathrm{eV}$ & & 6.2 & 7.62 & 8.51 & 7.25 & 6.94 & 10.5 & & 7.82 & & 8.84 \\
\hline$\varphi_{x}^{+}, \mathrm{eV}$ & 10.5 & 7.4 & 7.9 & 7.7 & 9.4 & 6.0 & 9.8 & 9.8 & 9.0 & 9.0 & 7.4 \\
\hline$M_{x}$, a.m.u. & 31.0 & 54.9 & 58.9 & 63.5 & 65.4 & 69.7 & 74.9 & 78.9 & 112.4 & 195.1 & 207.2 \\
\hline $\mathrm{RSC}_{\mathrm{IS}}^{\text {,theor }}$ & 1.45 & 1.72 & 1.90 & 2.05 & 2.10 & 1.91 & 2.21 & 2.51 & 2.75 & 2.07 & 2.91 \\
\hline $\mathrm{RSC}_{\mathrm{IS}}^{\text {,exper }}$ & 3.22 & 4.09 & 4.80 & 4.45 & 5.43 & 4.13 & 5.23 & 5.22 & 6.70 & 5.30 & 6.92 \\
\hline$\Delta R$ & 0.45 & 0.35 & 0.39 & 0.46 & 0.38 & 0.46 & 0.42 & 0.48 & 0.41 & 0.39 & 0.42
\end{tabular}

широко используют два способа: прямое разбавление и минерализацию путем кислотного разложения образцов в микроволновой (MW) приставке.

Прямое разбавление применяется в случае таких объектов, как цельная кровь, плазма крови и моча [19]. Поскольку кровь представляет собой сложную по составу биологическую жидкость с высоким содержанием как неорганических, так и органических веществ, для анализа цельной крови в ICP-MS в качестве пробоподготовки было использовано прямое разбавление.

Пробы цельной крови, поставленные в одноразовых вакуумных пробирках, хранились в морозильной камере при температуре $-18^{\circ} \mathrm{C}$. Перед пробопоготовкой их размораживали в течение нескольких часов до комнатной температуры и тщательно перемешивали для гомогенизации состава.

Раствор внутреннего стандарта (IS) с концентрацией $3 \mathrm{mg} / 1$ готовили путем разбавления с помощью 1 vol.\% $\mathrm{HNO}_{3}$ соответствующего одноэлементного стандарта $1000 \mathrm{mg} / \mathrm{l} \mathrm{Rh}$. Во всех исследуемых пробах концентрацию IS, равную $25 \mu \mathrm{g} / \mathrm{l}$, создавали путем добавления необходимого объема приготовленного раствора. Среди основных требований, предъявляемых к IS, следует отметить близость по физическим и химическим характеристикам IS и определяемых элементов, а также отсутствие спектральных наложений, влияющих на сигнал данного изотопа в рассматриваемой матрице. Для каждого из определяемых в биологических объектах элементов градуировки проводились в диапазоне концентраций, приведенных на сайте Независимой лаборатории ИНВИТРО с учетом конечного разбавления в процессе пробоподготовки.

При простом разбавлении жидких образцов цельной крови (сыворотки крови) к $0.5 \mathrm{ml}$ пробы добавляли $4.5 \mathrm{ml}$ разбавителя ( 1 vol.\% $\left.\mathrm{HNO}_{3}\right)$ и $25 \mu \mathrm{g} / 1$ раствора внутреннего стандарта. Для разбавления образцов мочи к $1.0 \mathrm{ml}$

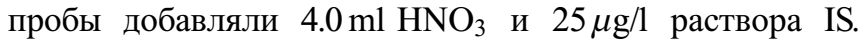
Перед анализом смеси тщательно перемешивали, затем центрифугировали до полного осаждения органических частиц.

Методика измерения. Для выяснения роли влияния кислоты на интенсивность аналитических сигналов в ряде работ (например, в [17]) используют несколько внутренних стандартов, связанных критерием атомной массы или потенциалом ионизации. В настоящей работе исследована роль матричного эффекта в устранении влияния кислоты с помощью одного IS, а именно родия $(\mathrm{Rh})$. Исследованию подвергались однократно заряженные ионы $\mathrm{Rh}^{+}$.

Как отмечено выше, были исследованы аналиты $\mathrm{Mn}$, $\mathrm{Co}, \mathrm{Cu}, \mathrm{Zn}, \mathrm{As}, \mathrm{Se}, \mathrm{Cd}, \mathrm{Pb}$ в образцах крови и мочи человека. Градуировочные растворы с концентрациями $0.5,1.0,5.0,50,100 \mu \mathrm{g} / 1$ готовили в $1 \mathrm{vol} . \% \mathrm{HNO}_{3}$ из многоэлементного стандартного раствора с концентрацией $10 \mu \mathrm{g} / 1$ ICP-MS-internal std mix part \# 5188-6525. В качестве матрицы сравнения, в среде которой были созданы градуировочные растворы, выступила 1 vol.\% азотная кислота.

Таким образом, для исследования в МС-ИСП с квадрупольным анализатором были заготовлены более 200 образцов крови и мочи, каждый из которых подвергался анализу при различных режимах работы прибора (мощности и частоты генератора, скорости потока аргона).

Влияние компонентов матрицы анализируемых растворов на величины интенсивностей сигналов исследуемых элементов оценивали следующим образом.

Согласно выражению $\mathrm{RSC}_{\mathrm{IS}}^{x}$ (1) с учетом (5), интенсивности абсолютного сигнала элемента $x$ по сравнению c IS в исследуемой матрице определяется

$$
\frac{J_{x}}{J_{\mathrm{IS}}}=\operatorname{RSC}_{\mathrm{IS}}^{x}\left(\frac{m_{x}}{m_{\mathrm{IS}}}\right)^{\gamma}
$$

Тогда отношения интенсивностей сигнала аналита и IS в исследуемой матрице 10 vol.\% $\mathrm{NHO}_{3}$ и матрицей сравнения ( 1 vol. $\left.\% \mathrm{HNO}_{3}\right)$ будет иметь вид соответственно

$$
\left(\frac{J_{x}}{J_{\mathrm{IS}}}\right)_{10 \%}=\exp \left(-\frac{\varphi_{x}^{+}-\varphi_{\mathrm{IS}}^{+}}{k T_{u}}\right)\left(\frac{m_{x}}{m_{\mathrm{IS}}}\right)^{\gamma}
$$

и

$$
\left(\frac{J_{x}}{J_{\mathrm{IS}}}\right)_{1 \%}=\exp \left(-\frac{\varphi_{x}^{+}-\varphi_{\mathrm{IS}}^{+}}{k T_{u}}\right)\left(\frac{m_{x}}{m_{\mathrm{IS}}}\right)^{\gamma},
$$


Таблица 2. Значение параметров работы прибора в „стандартном режиме“

\begin{tabular}{l|c}
\hline \multicolumn{1}{c|}{ Параметр } & Диапазон \\
\hline Мощность ВЧ генератора & $1400-1500 \mathrm{~W}$ \\
\hline Скорость потока аргона через распылитель & $1.15-1.251 / \mathrm{min}$ \\
\hline Глубина отбора пробы & $7.5-8.5 \mathrm{~mm}$ \\
\hline Потенциал на линзе экстрактора & $2-4 \mathrm{~V}$
\end{tabular}

Примечание. Были исследованы сигналы аналитов $\mathrm{Mn}, \mathrm{Co}, \mathrm{Cu}$, $\mathrm{Zn}$, As, $\mathrm{Se}, \mathrm{Cd}, \mathrm{Pb}$. В качестве IS применяли Rh. Градуировочные растворы с концентрациями элементов 1.0, 5.0, 20, 100, 400 и $1000 \mu \mathrm{g} / 1$ готовили в $1 \%$ vol. $\mathrm{HNO}_{3}$ из многоэлементного стандартного раствора $8500-694010 \mu \mathrm{g} / \mathrm{l}$. Для каждого определяемого элемента градуировку проводили в диапазоне концентраций,приведенных на сайте Независимой Лаборатории ИНВИТРО (табл. 3).

а разность этих отношений интенсивностей с учетом (7) будет

$$
\Delta R=\left[\left(\frac{J_{x}}{J_{\mathrm{IS}}}\right)_{10 \%}-\left(\frac{J_{x}}{J_{\mathrm{IS}}}\right)_{1 \%}\right] \exp \left(-\frac{\varphi_{x}^{x}-\varphi_{b}^{c}}{k T_{u}}\right)\left(\frac{m_{x}}{m_{\mathrm{IS}}}\right)^{0.6} .
$$

Отметим, что расчет $\mathrm{RSC}_{\mathrm{IS}}^{x}$, проведенный на основе (6) по экспериментальным данным, показал, что в пределах кислотности матрицы сравнения 1-10 vol.\% $\mathrm{HNO}_{3}$ для $\varphi_{\mathrm{IS}}^{+} \leq 9 \mathrm{eV} \gamma$ не превышает 0.6 .

Таким образом, количественное влияние компонентов матрицы анализируемых растворов на величины интенсивностей сигналов исследуемых элементов оценивали при помощи разности потенциалов ионизации и отношения атомных масс аналитов и IS.

Предварительные эксперименты были направлены, прежде всего, на выявление возможных, оказывающих влияние на сигналы, аналитов при матричных эффектах, и выбор подходящих методик анализа, представленных в литературе.

Настройку режима работы ICP-MS осуществляли с помощью программы „Autotune“ из пакета Chemstation, применяя стандартный раствор $10 \mu \mathrm{g} / \mathrm{Li}, \mathrm{Rh}, \mathrm{Y}, \mathrm{Ce}$ и Ti в $2 \%$ vol. $\mathrm{HNO}_{3}$. Программа, имеющаяся в пакете, обеспечивает максимальную чувствительность для режима $W=1400 \mathrm{~W}$ и скорости потока аргона через распылитель $v=1.201 / \mathrm{min}$. При этом степень образования двукратно заряженных ионов не превышала $2 \%$ (такой режим работы прибора называется „стандартным“ [2], табл. 2).

\section{Результаты и их обсуждение}

На рис. 1 представлены зависимости $\Delta R$ от мощности ВЧ-генератора. Концентрации компонентов, используемых для разбавления смесей, соответствовали усредненным значениям, встречающимся в литературе. Как видно из рисунка, при фиксированной скорости потока аргона $(v=1.21 / \mathrm{min}$ - оптимальной с точки зрения

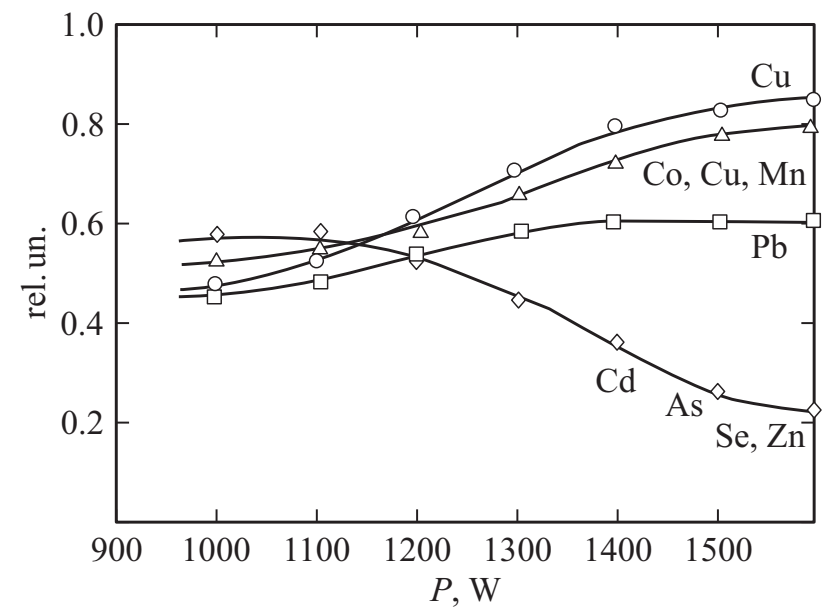

Рис. 1. Зависимость величины $\Delta R$ для элементов $\mathrm{P}, \mathrm{As}$ и $\mathrm{Se}$ от мощности генератора при фиксированной скорости потока аргона $1.201 / \mathrm{min}$.

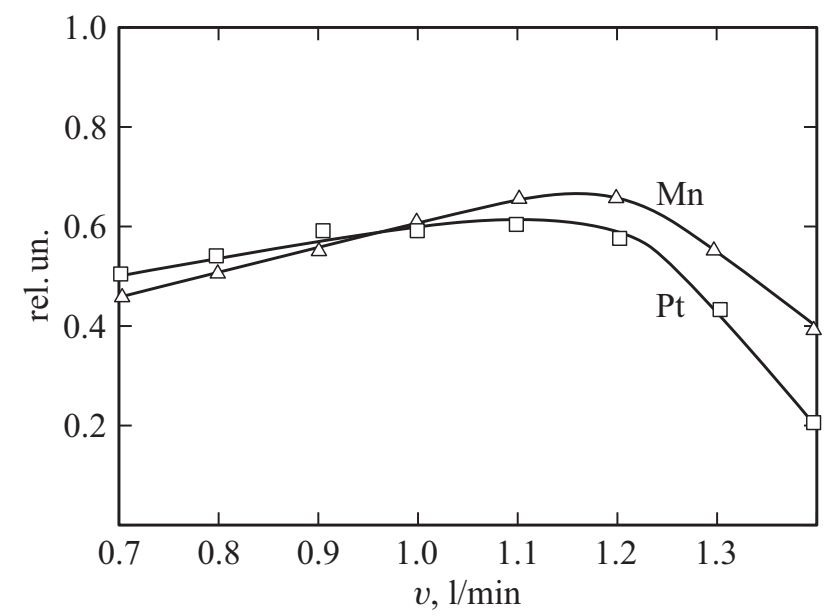

Рис. 2. Зависимость величины $\Delta R$ для элементов $\mathrm{Mn}$ и $\mathrm{Pt}$, зарегистрированных в среде $15 \mathrm{vol} . \% \mathrm{HNO}_{3}$, от скорости потока аргона через распылитель при мощности генератора $1750 \mathrm{~W}$.

чувствительности определения элементов в $2 \%$ азотной кислоте) уменьшение мощности генератора практически до нижнего предела $(1100 \mathrm{~W})$ позволяет несколько скомпенсировать рассматриваемый эффект для элементов с $\varphi_{i}^{+}<9 \mathrm{eV}$ ( $\left.\mathrm{Se}, \mathrm{Cd}, \mathrm{Co}\right)$ за счет, по-видимому, понижения температуры плазмы и, как следствие, уменьшения в ней концентрации ионов $\mathrm{C}^{+}$. Кстати, этот эффект характерен для некоторых элементов с высокими первыми потенциалами ионизации [20] таких, как $\mathrm{C}^{+}\left(\varphi_{x}^{+}=11.26 \mathrm{eV}\right)$, образующихся при вводе в плазму органического растворителя. Для элементов с $\varphi_{i}^{+} \geq 9 \mathrm{eV}$ подбор мощности не приводит к заметному изменению чувствительности. Она достигает наибольшего значения при $1450 \mathrm{~W}$.

Очевидно, что при простом разбавлении пробы следует учитывать влияние солей и органических веществ, присутствующих в биологических жидкостях. Кислотность анализируемых растворов при этом строго фиксирована за счет использования растворителя, содер- 
Таблица 3. Референсное содержание элементов в крови и моче, $\mu \mathrm{g} / 1$

\begin{tabular}{c|c|c|c|c|c|c|c|c}
\hline Образец & $\mathrm{Mn}$ & $\mathrm{Co}$ & $\mathrm{Cu}$ & $\mathrm{Zn}$ & $\mathrm{As}$ & $\mathrm{Se}$ & $\mathrm{Cd}$ & $\mathrm{Pb}$ \\
\hline Цельная кровь & $7-15$ & $0.5-2.5$ & $750-1500$ & $5500-9000$ & $0-10$ & $75-200$ & $\sim 5$ & $0-100$ \\
\hline Моча & $0.75-3$ & $0.25-2$ & $10-70$ & $200-1000$ & $0-350$ & $15-60$ & $0-1$ & $0-5$
\end{tabular}

Примеч ан и е. Для приготовления всех растворов использовали дэионизированную воду $(18.2 \mathrm{M} \Omega \cdot \mathrm{cm})$.

Таблица 4. Регистрируемые с помощью ICP-MS концентрации элементов в цельной крови в зависимости от количества анализов проб

\begin{tabular}{c|c|c|c|c|c|c|c|c|c}
\hline \multicolumn{10}{c}{ Концентрации, $\mu \mathrm{g} / 1$} \\
\hline $\begin{array}{c}\text { Количество } \\
\text { анализов }\end{array}$ & $\mathrm{Mn}$ & $\mathrm{Co}$ & $\mathrm{Ni}$ & $\mathrm{Cu}$ & $\mathrm{Zn}$ & $\mathrm{As}$ & $\mathrm{Se}$ & $\mathrm{Cd}$ & $\mathrm{Pb}$ \\
\hline 1 & 10.55 & 2.05 & 7.90 & 997 & 8400 & 80 & 170 & 5.0 & 80 \\
\hline 5 & 10.15 & 2.00 & 7.73 & 940 & 8200 & 77 & 160 & 4.7 & 77 \\
\hline 10 & 9.85 & 1.95 & 7.21 & 932 & 8110 & 72 & 151 & 4.7 & 72 \\
\hline 15 & 8.73 & 1.74 & 6.83 & 844 & 7450 & 69 & 145 & 4.5 & 70 \\
\hline 20 & 6.11 & 1.48 & 6.62 & 712 & 7120 & 62 & 138 & 4.1 & 65 \\
\hline 25 & 7.76 & 0.98 & 6.40 & 701 & 6672 & 58 & 120 & 3.8 & 65 \\
\hline 30 & 7.11 & 0.88 & 5.20 & 635 & 6221 & 54 & 108 & 3.7 & 48 \\
\hline 35 & 6.80 & 0.28 & 2.50 & 423 & 5500 & 50 & 97 & 2.5 & 42 \\
\hline 40 & 5.11 & 0.70 & 3.90 & 583 & 5200 & 51 & 91 & 2.0 & 40
\end{tabular}

жащего 1 vol.\% азотной кислоты. С другой стороны, поскольку содержание минеральных солей в цельной крови не превышает $0.9 \%$, а в моче - $2 \%$, их концентрации в растворе не могут быть выше 0.09 и $0.04 \%$ соответственно [21]. Сложнее дело обстоит с имитацией органической матрицы биожидкостей в случае цельной крови $(\sim 25 \mathrm{~g} / 1[22])$.

Таким образом, полного устранения не спектральных эффектов во всех рассматриваемых средах с помощью подбора мощности генератора добиться не удается, поэтому на следующем этапе при выбранном значении параметра и фиксированного режима работы массспектрометра мы изменяли скорость потока аргона через распылитель. С целью подтверждения необходимости именно понижения этой характеристики прибора ее изменяли как в сторону меньших, так и больших значений относительно оптимального (1.201/min).

Результаты исследований даны на рис. 2. Как видно из рисунка, при увеличении скорости потока аргона $1.41 / \mathrm{min}$ или его уменьшении до $10.8 \mathrm{l} / \mathrm{min}$ интенсивность абсолютных сигналов аналитов и IS снижается в среднем в 3 раза в зависимости от матрицы и определяемого изотопа.

Многочисленные эксперименты, проводимые с различными разбавителями $\left(\mathrm{HNO}_{3}, \mathrm{HCl}\right.$, глицин и карбомид), подтвердили тенденцию подавления аналити-
Таблица 5. Концентрации элементов в моче в зависимости от количества анализов проб

\begin{tabular}{c|c|c|c|c|c|c|c|c}
\hline \multicolumn{1}{c}{ Концентрации, $\mu \mathrm{g} / 1$} \\
$\begin{array}{c}\text { Количество } \\
\text { анализов }\end{array}$ & $\mathrm{Mn}$ & $\mathrm{Co}$ & $\mathrm{Ni}$ & $\mathrm{Cu}$ & $\mathrm{Zn}$ & $\mathrm{As}$ & $\mathrm{Se}$ & $\mathrm{Pb}$ \\
\hline 1 & 1.50 & 1.0 & 13 & 1100 & 870 & 270 & 40 & 3.0 \\
5 & 1.35 & 0.8 & 10 & 890 & 810 & 250 & 30 & 2.5 \\
10 & 1.27 & 0.73 & 9.3 & 673 & 765 & 245 & 27 & 2.3 \\
15 & 1.21 & 0.67 & 8.7 & 565 & 427 & 232 & 21 & 2.11 \\
20 & 1.16 & 0.55 & 8.1 & 570 & 401 & 217 & 18 & 1.89 \\
25 & 1.13 & 0.21 & 7.9 & 520 & 387 & 205 & 16 & 1.82 \\
30 & 1.10 & 0.13 & 7.0 & 500 & 361 & 185 & 15 & 1.79 \\
35 & 0.93 & - & 4.0 & 400 & 287 & 162 & 12 & 1.60 \\
40 & 0.75 & - & 6.1 & 490 & 311 & 155 & 15 & 1.65
\end{tabular}

ческих сигналов на выходе прибора. В обоих случаях (увеличение или уменьшение $v$ ) решающую роль играет температура плазмы: при увеличении скорости аргона температура понижается за счет менее эффективной ионизации и большей загрузки компонентами анализируемого раствора, а при медленной подаче пробы, очевидно, просто снижается скорость транспортировки аналита.

Для нивелирования влияния матричных эффектов на интенсивность сигналов $(\Delta R)$, по-видимому, необходимо уменьшить скорость аргона через распылитель. Объяснением хода кривых на рис. 2 могут быть повышение температуры за счет меньшей загрузки компонентами матрицы, а также увеличение времени нахождения проб в высокотемпературной зоне за счет смещения ее в сторону инжектора горелки.

Одной из причин снижения интенсивности сигналов аналитов на выходе может быть искажение расчетной траектории ионов после выхода их из плазмы. Причиной же искажения, по-видимому, является диэлектрическое покрытие, образующееся в результате оседания положительных ионов солей и органических соединений растворителей и органических веществ, присутствующих в биожидкостях, на конструкционные элементы прибора. Действительно, ионы, попадая на поверхности электродов, задающих форму поля, нейтрализуются и создают диэлектрическое покрытие на этой поверхности (скиммера, самплера). Расчеты показывают, что уже после нескольких десятков анализов толщина диэлектрического покрытия может достигать $10^{-4} \mathrm{~cm}$. Ионы, попадая на эту поверхность, заряжают ее и создают 
дополнительное электрическое поле, которое может искажать траекторию ионов аналитов. Встречаются работы, где исследуется возможность снижения МЭ за счет настройки ионной оптики [23].

Для проверки этой версии нами были исследованы масс-спектры цельной крови (табл. 4) и мочи (табл. 5) при одинаковых условиях пробоподготовки через каждые 5 анализов. Как видно из таблиц, концентрации обоих образцов изменяются с увеличением количества анализов, и после 40 анализов концентрации ионов уменьшаются вдвое. При этом если для легких элементов $(\mathrm{Mn}, \mathrm{Cu}, \mathrm{Co})$ падение чувствительности с увеличением числа импульсов можно объяснить увеличением искажающего поля, то для тяжелых элементов этого утверждать нельзя. Вполне вероятно, что уменьшение интенсивности ионов (As, Se, Pb) связано с наличием двух и более кратно заряженных ионов этих элементов.

\section{Выводы}

Исследован и количественно оценен вклад неспектрального матричного эффекта в искажении результатов анализа разбавленных биологических жидкостей. Показано, что влияние солевой, органической и кислотной матриц на результаты анализа носит аддитивный характер, т.е. она зависит от метода пробоподготовки, выбора растворителя и режима работы прибора. Изучена возможность устранения данного эффекта за счет настройки параметров прибора. Показано, что основной вклад в уменьшение влияния матричного эффекта для большинства элементов ( $\mathrm{Se}, \mathrm{As}, \mathrm{Pb}$ ) вносит повышение мощности генератора и снижение скорости потока аргона через распылитель. При этом наибольшее значение чувствительности для ионов с $\varphi_{i}^{+}<9 \mathrm{eV}$ достигает при мощности генератора $400 \mathrm{~W}$ и скорости аргона $1.21 / \mathrm{min}$, а для ионов с $\varphi_{i}^{+}>9 \mathrm{eV}$ оптимальным считается $1100 \mathrm{~W}$.

\section{Список литературы}

[1] Пупышев А.А., Суриков В.T. Масс-спектрометрия с индуктивно связанной плазмой. Образование ионов. Е.: УрО РАН. 2006. $276 \mathrm{c}$.

[2] Agilent 7700 ICP-MS Specification Agilent Technologies 2012 USA G127 127-4254E

[3] Parsons P.J., Barbosa Jr.F. // Spectrochim. Acta Part B. 2007. Vol. 62. N 9. P. 992-1003.

[4] Ярошенко Д.В., Кариова Л.А. // Журн. аналит. хим. 2014 T. 69. № 4. C. 351-358.

[5] Xu R., Fan L., Rieser M., El-Shourbagy T. // J. Pharm. Biomed. 2007. Vol.44. P. 342-355.

[6] Ciric A., Prosen H., Jelikic-Stankov M., Durdevic P. // Evaluation of matrix effect in determination of some bioflavonoids in foods amples by LC-MS/MS method. Talanta. 2012. Vol. 99. P. 780-790.

[7] King R., Bonfiglio R., Fernandez-Metzler C., Miller-Stein C., Olah T. // J. Am. Soc. Mass Spectrom. 2000. Vol. 11. N 11. P. $942-950$.
[8] Dams R., Huestis M., Lambert W., Murphy C. // J. Am. Soc. Mass Spectrom. 2003. Vol. 14. P. 1290.

[9] Stewart I.I., Olesik J.W. // J. Anal. At. Spectrom. 1998. Vol. 13. N 12. P. 1313-1320.

[10] Todolí J.-L., Mermet J.-M. // Spectrochim. Acta Part B. 1999. Vol. 54. N 6. P. 895-929.

[11] Карташева М.А., Боровицкий В.Г., Дубников С.Ю. // Патент РФ. № 2039970 класс G01 № 12172.

[12] May T.W., Wiedmeyer R.H. // Atom. Spectrosc. 1998. Vol. 19. P. $150-155$.

[13] Нурубейли Т.К., Нурубейли З.К., Нуриев К.З., Гурбанов К.Б. // ЖТФ. 2017. Т. 87. Вып. 2. С. 277281. [Nurubeyli T.K., Nurubeyli Z.K., Nuriyev K.Z., Gurbanov K.B. // Techn. Phys. 2017. Vol. 62. N 2. P. 305 309.]

[14] Рамендик Г.И., Фатюшина Е.В., Степанов А.И. // Журн. аналит. Химии. 2001. Т. 56. Вып. 6. С. 561-573.

[15] Нурубейли 3.К., Гашимов А.М., Манучар С.А., Нуриев К.З., Гурбанов К.Б. // Электронная обработка материалов. 2008. № 2. С. 98-105.

[16] Нурубейли 3.К., Нуриев К.З., Керимов Г.М. // Электронная обработка материалов. 2013. Т. 49. № 4. С. 68-73.

[17] Пупышев А.Ф., Семенова Е.В. // Аналитика и контроль. 2000. T. 4. № 2. C. 120-140.

[18] Эмсли Дж. Элементы / Пер. с английского М.: Мир, 1993. $256 \mathrm{c}$.

[19] Tanaselia C., Frentiu T., Ursu M., Vlad M., Chintoanu M., Cordos E., David L., Paul M., Gomoiescu D. // Adv. Mat. 2008. Vol. 2. N 2. P. 99-107.

[20] Lide D.R., Raton B. CRC Handbook of Chemistry and Physics, 89th edition. UK: CRC Press, 2008. 2736 p.

[21] Awad S., Allison S.P., Lobo D.N. // Clin. Nutr. 2008. Vol. 27. N 2. P. $179-188$.

[22] Скальный А.В. Химические элементы в физиологии и экологии человека. М.: Оникс 21 век, 2004. 216 с.

[23] Карандашев В.К., Лейкин А.Ю., Жерноклеева К.З. // Журн. аналит. хим. 2014. Т. 69. № 1. С. 26. 